\title{
FUENTES FISCALES E HISTORIA AGRARIA. EL DEBATE EN TORNO DE LAS POSIBILIDADES HEURÍSTICAS DE LOS AMILLARAMIENTOS
}

\author{
POR \\ M. ${ }^{\text {a }}$ TERESA PÉREZ PICAZO
}

El centenario de la reforma fiscal de Mon-Santillán generó la publicación de numerosos trabajos a lo largo del año 1996. Elaborados unas veces desde el punto de vista de la técnica tributaria y otros desde una perspectiva histórica, sus autores llegaron a idéntica conclusión: la importancia del impacto ejercido por la reforma en cuestión sobre la evolución de la fiscalidad española contemporánea. El presente artículo, ubicado en el segundo de los campos citados, tiene como objeto una breve reflexión sobre las posibilidades que ofrece como fuente histórica la documentación generada por una de las figuras tributarias surgidas en 1845: la inicialmente denominada Contribución de Inmuebles, Cultivo y Ganadería que acabaría siendo conocida como Contribución Territorial. El tema ha sido objeto de cierto debate a partir de los bien documentados trabajos de J. Pro Ruiz (1992, 1993, 1994 a y b, 1996), en los que se reiteran una y otra vez las deficiencias heurísticas de dichos textos. En fechas recientes se expresaba como sigue «...ciertos historiadores del siglo xx... se han lanzado a sustituir la realidad por un documento de ficción, reconstruyendo una imagen falsa de la sociedad decimonónica a partir de datos que, como hemos dicho, resultan del regateo político y no de una averiguación rigurosa.

M. ${ }^{a}$ Teresa Pérez Picazo: Departamento de Sociología e Historia Económica, Universidad de Murcia.

Estudios Geográficos Tomo LIX, n. ${ }^{\circ} 231$, abril-junio 
Así se escribe la historia» (1996, p. 130). Salta a la vista la dureza de las críticas vertidas.

Y, sin embargo, el análisis en profundidad de esta documentación tan denostada revela que, como casi siempre, las descalificaciones rotundas no suelen ajustarse demasiado bien a la realidad que pretenden describir. En tal sentido apuntan las investigaciones verificadas a escala local o regional por un amplio colectivo de geógrafos e historiadores en este último decenio, en las que se recurre sistemáticamente a los Amillaramientos y Cartillas Evaluatorias. Investigaciones que han alcanzado especial relieve en los casos de Andalucía Occidental (Cruz Villalón, J.: 1980; Mata Olmo, R.: 1987)) y Oriental (Martínez Marín, M.: 1995), Cataluña (equipo reunido en torno a R. Garrabou: (coord.): 1992; Segura, A.: 1993), Extremadura (Sánchez Marroyo, F.: 1993), Galicia (Villares, R.: 1982), Mallorca (Suau, F.: 1991), Navarra (Grupo de Investigación del Instituto Gerónimo de Uztáriz: 1992) y Valencia (Obiol Menero, E.: 1988; Romero González, J.: 1983). El reducido universo de análisis de estos trabajos ha permitido a sus autores no sólo depurar cuidadosamente los datos y contrastarlos con otros de distinto origen sino, lo que es más importante, descubrir nuevas formas de utilización de los mismos. Mi propia investigación sobre la región murciana apunta en el mismo sentido. Por consiguiente, en las páginas que siguen me ocuparé, primero, de las deficiencias reales de las precitadas fuentes, derivadas en gran parte de su origen fiscal y de las características del gravamen que las produjo. Y, después, de los sistemas a seguir para obviar - siquiera sea parcialmente- dichas dificultades, amén de sugerir nuevas vías de aproximación a unos textos preestadísticos que en amplia medida siguen desaprovechados.

\section{Una contribución de «producto» recaudada} por el sistema de cupo

Aunque se ha escrito suficientemente sobre los objetivos, significado y logros de la reforma de Alejandro Mon (Artola, M.: 1986; Comín, F.: 1988; Fontana, J.: 1977; Fuentes Quintana, E.: 1961, 1990; Segura, A.: 1988), convendría empezar recordando que en el terreno de la teoría estuvo inspirada por los principios liberales concernientes a la fiscalidad: universalidad, igualdad de todos los ciudadanos ante el fisco y prefe- 
rencia por las cargas directas. La aplicación de tales principios, sin embargo, no iba a ser cosa fácil debido al peso político de las «clases propietarias» en el nuevo estado liberal. De ahí que el proceso de cambio abierto en 1845 fuera cualquier cosa menos rápido, y que la pieza fundamental de la Ley de Presupuestos votada el 23 de mayo de 1945, la Contribución de Inmuebles, Cultivo y Ganadería, se encontrara con numerosas dificultades prácticas. Y ello pese a que constituía la plasmación de las ideas fiscales del liberalismo, y a que hubiera debido permitir la extensión de la imposición directa sobre la mayor parte del territorio nacional, sustituyendo así a los antiguos diezmos percibidos por la Iglesia.

En efecto, la aplicación de la nueva carga tributaria tropezó desde el primer momento con un doble escollo: la concepción de la misma como un impuesto de cupo y el hecho de que la detracción debiera hacerse sobre el producto de las fincas en un contexto caracterizado por la pobreza de la información estadística. Comenzando así por el problema del cupo, se trataba de repartir el líquido total a percibir, estipulado por el Estado, utilizando tres instancias de poder sucesivas en la jerarquía institucional: el Congreso de los Diputados - aprobación de la cifra global y distribución de la misma entre las provincias (cupos provinciales)-, Diputaciones - reparto entre los municipios-y Ayuntamientos -reparto entre los vecinos contribuyentes. Todos los especialistas del tema concuerdan en señalar que las cantidades a las que se llegaba en estos tres niveles no eran el resultado de las desigualdades económicas reales entre las distintas circunscripciones sino de la pugna entre sus representantes en el primero, del peso de las coaliciones políticas y las redes de influencia en el segundo (Laguna, A. y Martínez Gallego, F.: 1995) y de la mayor o menor fuerza de las oligarquías locales en el tercero. Obviamente, la falta de un Catastro o de unas estadísticas mínimamente fiables sobre la geografía económica del país agravaba la situación.

Tomamos así contacto con el segundo de los problemas citados, la carencia de fuentes catastrales, imprescindibles para el buen funcionamiento de una contribución de producto, de por sí difícil de percibir. España fue uno de los pocos países europeos que no llegó a contar con un Catastro en el cual basar la recaudación de su contribución territorial hasta mediados del siglo xx. Ello privó a la Hacienda Pública de la posibilidad de conocer y actualizar la base imponible del tributo di- 
recto más importante y, por consiguiente, de incrementar su recaudación; sin olvidar que tal desconocimiento permitió la existencia de graves injusticias y desigualdades regionales e individuales en el reparto de la carga. El hecho no resulta difícil de explicar si se recuerda una vez más el gran peso político de los terratenientes, los cuales temían que el Catastro pusiese al descubierto su verdadera capacidad de pago, prefiriendo en consecuencia políticas de aumento de los impuestos indirectos. La falta de recursos financieros de los sucesivos gobiernos hizo el resto.

En estas condiciones, el instrumento elegido para poder concretar en el terreno administrativo el recién creado impuesto fueron los llamados Padrones Generales de la Riqueza Inmueble (R.O. del 23-V-1845 y del 6-XII-1845), que se elaboraron a partir de las declaraciones juradas de los contribuyentes, municipio por municipio. En un primer momento, Alejandro Mon intentó aplicar un método eficaz para recabar la información necesaria. Se trataba de elaborar una Estadística de la Riqueza, destinada a complementar la reforma, cuya ejecución se atribuyó a una $D i$ rección General de Estadística de nueva creación, que organizaría los trabajos necesarios para la confección de una especie de Catastro estimativo, inspirado en el modelo napoleónico de grandes «masas de cultivo» - 1801 a 1805-. El organismo en cuestión estaba representado en cada capital provincial por un Comisionado de Estadística, es decir, por un funcionario del gobierno independiente de los intereses locales, el cual, ayudado por algunos técnicos, podría corregir las estimaciones recibidas y comprobar las declaraciones juradas. Huelga decir que el procedimiento no gustó a las oligarquías de propietarios, lo que explica el bloqueo del proyecto como consecuencia del olvido (?) de los legisladores, que omitieron asignar en el presupuesto las dotaciones necesarias para llevarlo a término.

De esta forma, la recogida de datos quedó definitivamente en manos de las instancias locales a través de las Juntas Periciales, complementadas a nivel provincial por las Comisiones de Evaluación y Repartimiento. Las primeras, que son las que aquí nos interesan, estaban integradas por los miembros del Ayuntamiento y por un número igual de «peritos repartidores», la mitad designados por el mismo y la otra mitad por Hacienda sobre una lista triple de contribuyentes que elaboraba el propio municipio, cuyo Alcalde las presidía siempre. En definitiva, se trataba de una representación de la oligarquía de la localidad cuyos miem- 
bros pactaban entre sí la forma de distribuir el nuevo impuesto directo de la forma más ventajosa posible para sus intereses y sin la presencia de los propietarios modestos.

En 1850 el sistema de Padrones de riqueza fue sustituido por un tipo distinto de documento llamado a perdurar durante el resto del siglo XIX y la primera mitad del XX: los denominados Amillaramientos de la riqueza individual contribuyente (Circular del 7-V-1850 y R.O. del 9-XI1853). No está de más señalar, sin embargo, que en los primeros años que siguieron a esta disposición pervive todavía en algunas zonas el término «padrón», legalmente ya prescrito. Se les puede describir como un listado literal de los propietarios y de las fincas rústicas y urbanas que poseían cada uno de ellos, sin que precediera deslinde de las parcelas, levantamiento de planos, medición del territorio o prueba pericial alguna para estimar su valor y rendimiento; de ahí las justificadas críticas sobre el escaso grado de fiabilidad de muchos de ellos. Por último, la Real Orden de 1853 antes aludida declaró taxativamente la perpetuidad de los nuevos textos y, asimismo, la obligación de constituir anualmente un apéndice en el que constaran los movimientos que la propiedad hubiera experimentado. Pese a ello, sólo se dispuso de forma general la rectificación de los mismos en dos ocasiones, los años 1860 y 1885, en este último como desarrollo de lo establecido por la ley y reglamento de contribución territorial que acababa de ser promulgada. Por tal razón suelen designarse con el apelativo de Refundición del amillaramiento, pues el citado texto legal establecía que se procediera a la fusión en un mismo documento de los amillaramientos y apéndices que rigieran en aquella fecha.

Concluyendo, a primera vista resulta innegable que un sistema como el descrito facilitaba el fraude, la ocultación y el desigual reparto de los cupos. Tanto más cuanto que, aunque la ley concedería a los vecinos la posibilidad de reclamar contra la forma en que se había hecho la distribución, las características del mundo rural en la mayor parte de España durante el siglo XIX impedía a los campesinos luchar contra este estado de cosas. En unos campos caracterizados por la superpoblación relativa y el «hambre de tierra», era difícil que los labradores osaran enfrentarse con el colectivo propietario, ante el temor de que no les renovasen los contratos o les subiesen la renta en un mercado de la tierra tan presionado por el lado de la demanda. Además, cuando se producían reclamaciones, el procedimiento administrativo era tan riguroso 
que exigía una nueva evaluación de la riqueza por los funcionarios de Hacienda, lo cual no convenía a nadie, de ahí que se evitaran lo más posible. A ello contribuía la elite terrateniente que se presentaba ante sus convecinos como el portavoz de los intereses de todos para limitar los «abusos» del poder central. Ese caldo de cultivo permitiría años después la emergencia de los caciques.

\section{Aspectos técnicos de los Amillaramientos}

Llegados a este punto y conocidos ya los objetivos, agentes intervinientes y disposiciones legales de mayor interés, procede el comentario sobre los aspectos puramente técnicos de la fuente. Recordemos, para empezar, que el proceso se iniciaba con la redacción de la Cartilla Evaluatoria por los peritos repartidores, los cuales dividían las tierras del municipio en clases (regadío, secano, viña, olivares, frutales, bosque, erial, etc.) y calidades (cuatro), y posteriormente calculaban, sobre la base de los últimos ocho o diez años el producto y los gastos de explotación medios por unidad de superficie que correspondían a cada clase y calidad de tierra. De la diferencia entre producto y gastos surgía el líquido imponible por unidad de la misma en función obviamente de su clase y calidad. Por último, de la aplicación de estos cálculos a las diferentes fincas que, según las declaraciones juradas poseía cada propietario, resultaba el total a pagar por cada uno de ellos una vez que la Junta Pericial hubiera establecido la cuota impositiva, parte alícuota del cupo asignado al municipio. Conviene tal vez advertir, para terminar, que las Cartillas de Evaluación - documento precioso para la historia de la explotación agraria - presentan un problema: la falta de actualización por campaña de los distintos productos líquidos de los cultivos según la marcha anual de las partidas de gastos e ingresos. Sólo en dos ocasiones se modificaron, en 1860 y en 1886 , por lo que resulta prácticamente imposible proceder mediante ellas a estudios locales de coyuntura de las distintas variables que figuran en sus páginas (precios, salarios, costes de producción en general, rentas de la tierra, etcétera).

Pasando a los amillaramientos propiamente dichos, la información que proporcionan puede ser general e individual (Segura, A.: 1988). En lo que se refiere a la primera se trata del año de confección del texto, la composición del Ayuntamiento y la Junta Pericial, la Cartilla Evaluatoria, 
las medidas de extensión agraria utilizadas y un resumen final. En este último figuran los totales de tierra de cada clase y calidad, así como los relativos a las fincas urbanas, las cabezas de ganado de cada especie y el número de propietarios de los distintos tipos de fincas. Para cada uno de estos epígrafes y sus subdivisiones se da el Producto Total, las Bajas de Cultivo o de Explotación y el Producto Líquido resultante de la diferencia entre el monto alcanzado por los dos conceptos anteriores.

En cuanto a la información individual suele figurar el nombre y apellidos del propietarios (distinguiendo entre vecinos y forasteros); las parcelas que posee cada uno, especificando la extensión, calidad y clases de cultivo, las cabezas de ganado y las casas de labor y/o de ubicación urbana. En ocasiones - no siempre - figura en el texto la relación de colonos, que puede presentarse de dos formas: bien después de cada propietario, añadiendo al lado del nombre de cada uno de ellos las tierras que trabajan y la forma de tenencia, bien al final, figurando entonces la lista nominativa de explotadores indirectos e indicando por supuesto las parcelas cultivadas por ellos y el propietario al que pertenecen.

A partir de la Real Orden, ya citada, de 1853, se enriqueció este capítulo informativo con la inclusión de la distribución del producto líquido entre colono y propietario. Así quedaba constancia de la parte del excedente que el segundo retenía en concepto de renta y de aquella otra que iba a parar a las manos del primero como cultivador directo. En opinión de Mata Olmo (que ha trabajado sobre la Campiña andaluza) cuando se cotejan los datos procedentes de los amillaramientos con los proporcionados por las actas notariales se comprueba que las cantidades de renta atribuidas a las grandes fincas consignadas en los primeros son, en la práctica totalidad, idénticas a las que constan en escrituras públicas de arriendo (Mata Olmo, R. y Romero González, J.: 1988, p. 240). En algunos municipios del Noroeste murciano - Caravaca, Moratalla - y del Campo de Cartagena - San Javier, Torre Pacheco, Fuente Alamo- he apreciado las mismas coincidencias. A la vista de ello resultan, pues, factibles los cálculos sobre la distribución del excedente neto o plusvalía entre propietarios rentistas y labradores arrendatarios (Pérez Picazo, M.T.: 1991).

De lo dicho se deduce que la masa de datos proporcionada por estos textos documentales es impresionante. Existe, sin embargo, tal desconfianza hacia la fiabilidad de la fuente que durante muchos años los especialistas de historia agraria optaron por su no utilización, como pre- 
conizaba el propio Tuñón de Lara. Las principales limitaciones al respecto pueden reunirse como sigue:

1. ${ }^{\circ}$ La ocultación superficial pura y simple, que constituye el defecto más conocido y criticado. Es bien sabido, por ejemplo, que a la altura de 1879 el nivel de la misma suponía el 40\% de la tierra a escala nacional, mientras que en las distintas provincias se encontraban cifras superiores al $60 \%$ e incluso del $80 \%$ en ciertas zonas montañosas. No obstante, existen procedimientos o soluciones para lograr una estimación adecuada; así, se puede contrastar la información contenida en apeos y amillaramientos con la proporcionada por las imágenes cartográficas coetáneas y, a su vez, estos datos se pueden cotejar con los resultados aportados por los Catastros y mapas parcelarios del siglo xx. Ahora bien, la diversidad al respecto es tan grande que el valor de los precitados textos en los distintos espacios geográficos de la Península oscila entre límites muy amplios, de ahí que difícilmente puedan deducirse unas conclusiones de tipo general. En Galicia, por ejemplo, R. Villares nos informa de la situación lastimosa de la fuente en las montañas de Lugo, atribuible a la actitud negativa de los perceptores de renta y los campesinos: en el quinquenio 1850-1855 solo una tercera parte de los Ayuntamientos de dicha provincia remitieron a las oficinas de Hacienda los amillaramientos y, a partir de dicha fecha, ya no se encuentran otros hasta mediados del siglo Xx. El panorama cambia completamente en las regiones de Valencia y Murcia (Calatayud, S.: 1989; Grupo de Historia Agraria de Murcia: 1992) gracias a la presencia de dos circunstancias concomitantes: el predominio de la pequeña explotación campesina y de la gestión indirecta, por un lado, y la existencia de numerosas zonas de regadío, objeto desde la Baja Edad Media de reiteradas y minuciosas mediciones superficiales recogidas en los sucesivos $\mathrm{Pa}$ drones de Acequias. Ello dificultaba enormemente toda pretensión de alterar la realidad de manera sustancial. El hecho se repite en Mallorca (Suau, J.: 1991), donde el nivel de ocultación se ha calculado en un $4,01 \%$, y en los regadíos navarros.

Más paradójico resulta lo sucedido en el Valle del Guadalquivir, donde Mata Olmo señala que la superficie poseída y declarada por los grandes propietarios en el amplio término de Córdoba presenta prácticamente la misma extensión en el Catastro de Ensenada y en el Amillaramiento de 1860; el geógrafo andaluz ha podido constatar, además, para una muestra de cortijos, la correspondencia de superficie entre la 
fuente fiscal en cuestión y las escrituras públicas (Mata Olmo, R.: 1987). $\mathrm{Y}$ otro tanto se detecta en lo relativo a las propiedades de los duques de Osuna y Medinaceli, repartidas en distintos municipios de la Depresión Bética. Bernal, hace cierto tiempo, atribuyó el hecho a que desde los primeros amillaramientos la nobleza andaluza procuró registrar minuciosamente todas sus fincas debido al cuestionamiento de su status territorial en la etapa de consolidación del liberalismo (Bernal, A.M. y Drain, M.: 1975).

2. ${ }^{\circ}$ La ocultación relativa, es decir, la infravaloración de superficies de los aprovechamientos de mayor riqueza y, dentro de los mismos, de las tierras de mejor calidad. Lógicamente, en este segundo aspecto las diferencias provinciales y municipales son aun mayores. Así, en Cataluña, la expansión del viñedo se registra con relativa fidelidad en unos casos y con muy poca en otros (Ferrer, Ll.; Segura, A.; Suau, J.: 1985; Ferrer; Ll: 1987; Saguer, E.: 1992), en Mallorca dicha expansión resulta fácilmente detectable en las pequeñas explotaciones, pero no en las grandes (Suau, J. 1991, Op.Cit.) y en Murcia sucede otro tanto en los municipios de Yecla y Jumilla, donde se desarrolló en la segunda mitad del siglo XIX una zona de monocultivo vinícola. Fenómeno este último bastante generalizable, ya que el fraude suele ser mayor en el caso de los terratenientes de cierta consideración, en cuyas declaraciones se percibe que la calidad media de las tierras está siempre por debajo de la confesada por los propietarios modestos y, en consecuencia, el porcentaje impositivo resulta ser inversamente proporcional a la cantidad de tierra poseída. En el mismo sentido apuntaría, según J. Pro, la inexistencia de una correlación significativa entre los porcentajes de las superficies no declaradas y cualquiera de los indicadores referentes al grado de concentración de la propiedad, aserto este último desmentido por minuciosos estudios de Ll. Ferrer; R. Mata Olmo; J. Suau y A. Segura ya citados

3. ${ }^{\circ}$ La falta de homogeneización de criterios cuando se confeccionaron los Amillaramientos. Así, las Juntas Periciales solían utilizar las medidas de extensión agraria que se aplicaban en cada municipio, las cuales eran extraordinariamente diversas. La solución habría sido difundir con mayor rapidez el uso del sistema métrico decimal, que comienza a imponerse desde el gobierno central a partir de 1849, con el fin de eliminar la diversidad en cuestión. Pero el éxito conseguido al respecto fue escaso, como lo revela el hecho de que las viejas medidas si-

$$
-293-
$$


guieron utilizándose en el campo hasta el primer tercio del siglo xx. En algunos casos, la administración recurrió a artilugios simplificadores como el de atribuir a la fanega una difusión generalizada y, lo que es más grave, asignarle un número fijo de hectáreas en cada provincia. En la realidad, cada comarca y a veces cada municipio tenía su fanega particular e incluso podía ocurrir dentro de la misma localidad que la extensión fuera diferente en el secano y en el regadío, como sucedía en Murcia donde, por ejemplo, el municipio de Caravaca poseía, además de la fanega de secano $(0,66 \mathrm{ha})$, y de regadío ( $0,33 \mathrm{ha})$, una tercera correspondiente al regadío «estercolado» $(0,11 \mathrm{ha})$. Pero este falso «reinado» de la unidad de superficie castellana no pudo evitar la aparición en los amillaramientos de medidas como los almudes albacetenses, las aranzadas andaluzas, las tahúllas murcianas, las hanegadas valencianas, los ferrados gallegos, etc., etc. Disparidades a las que hay que añadir todavía las resultantes del empleo de denominaciones distintas para referirse a las mismas clases de cultivo - el maíz se llama a veces panizo y las patatas criadillas- o, lo que es peor, del mismo término con distinto sentido. A veces, incluso, el uso de una denominación no implica la misma valoración en todos los casos: el producto líquido final sobre el que se aplica la cuota contributiva de una hectárea de secano o de regadío, de trigo o de viña, puede oscilar entre límites relativamente amplios.

Este conjunto de prácticas ha inclinado a J. Pro a ir más allá de la pura crítica cuantitativa que desarrolló en sus primeros trabajos; rechazando el uso de los Amillaramientos por razones más de fondo. En su opinión, dado el poder de las oligarquías locales su confección sólo podía responder a una apariencia de averiguación, porque averiguación verdadera de la distribución de la propiedad no la había ni la intención de hacerla posible tampoco; por consiguiente, los precitados documentos no eran otra cosa que «un espacio para la negociación y una negociación que se producía en cascada» (Pro Ruiz, J.: 1996). Aun estando de acuerdo con esta afirmación, parece sorprendente que su autor no se percate de la importancia del hecho de que puedan utilizarse los mencionados textos como indicador del peso efectivo de las estructuras oligárquicas y caciquiles. Ello lo han percibido muy bien, en cambio, el equipo de historiadores que trabaja en Granada en torno a M. Rodríguez de Molina (1992, 1993 a y b, 1995) sobre las funciones económicas de los poderes locales a lo largo del siglo XIx; las distintas publicaciones que han ido 
apareciendo han mostrado cómo dichos poderes tenían asignada una tarea fundamental, consistente en asegurar la reproducción de las condiciones de producción existentes. Claro está que el reparto de la carga fiscal constituía un elemento fundamental del proceso.

En segundo lugar, la negativa contra el uso heurístico de la documentación analizada se apoyaría en otro argumento de carácter a la vez antropológico y social. Se trata de la consideración del propio texto del amillaramiento como el resultado de una racionalidad, la campesina, profundamente opuesta a la del estado burgués (Pro Ruiz, J.: 1994). De nuevo coincido en términos generales con la idea, pero de nuevo disiento en que el fenómeno reste validez al texto en cuestión ya que, por el contrario, abre un nuevo campo de posibles aprovechamientos en el sentido de ayudarnos a aprehender la fisonomía de unas agriculturas de dominante campesina ampliamente hegemónicas en la España rural durante la mayor parte de la centuria estudiada (Pérez Picazo, M.T.: 1995a). Recuérdese lo dicho anteriormente sobre la difícil difusión del sistema métrico, a lo que podría añadirse el mantenimiento de ciertos aprovechamientos comunales - que a veces figuran en los amillaramientos- $-\mathrm{y}$, en otro orden de cosas, la pervivencia de las subdivisiones generadas por el sistema de rotación trienal (las «hazas»y «hojas») en el secano y de la asignación centralizada del agua a la tierra en los regadíos. En estos últimos, el indicado sistema generaba una estricta división del espacio en función de los caudales a los que tenían acceso las distintas zonas regadas, cuya distinta denominación aparece con frecuencia. Es el caso de los regadíos de fuente de todo el Sureste, como se detecta, por ejemplo, en Yecla, donde la superficie regada se dividía en seis sectores cuyas asignaciones no podían ser alterados: huertas, huertos, baños, viñas, viñales y hondo del campo ${ }^{1}$. El hecho se repite, con otra nomenclatura como es obvio, en la mayor parte de estas pequeñas huertas, cuya complicada organización, muchas veces de raigambre medieval, puede seguirse en muchas ocasiones trabajando con asiduidad sobre los amillaramientos correspondientes.

${ }^{1}$ En la región murciana se conservan los Amillaramientos de 1860 en casi todos los municipios, con la excepción señalada de los tres mayores -Murcia, Cartagena y Lorca-. Destacan por su fiabilidad y correcta ejecución los de Alhama, Cehegín, Fortuna, Jumilla, Moratalla, Mula, Totana y Yecla, todos ellos conservados en los Archivos $\mathrm{Mu}$ nicipales respectivos. En la actualidad el rico material que encierran sus páginas ha sido informatizado y sometido a crítica por los miembros de un equipo de investigación dirigido por M. ${ }^{\mathrm{a}}$ Teresa Pérez Picazo. Ver la bibliografía citada al final. 
La serie de hechos que acabamos de enumerar puede y debe interpretarse en el sentido de aceptar la fuerte vitalidad de la comunidad local como instancia de base de la sociedad rural, lo que supone, a su vez, asumir el importante peso de los vínculos familiares y de vecindad o de las redes clientelares. En la cuestión que aquí nos interesa todo ello se tradujo en la idea de que lo esencial era evaluar la riqueza de los pueblos y no la de cada vecino; huelga decir que ello convenía perfectamente a los intereses de las oligarquías locales de propietarios. Así se explica que, aparte de las razones ya expuestas, se tolerara sin gran resistencia el que las autoridades municipales dijeran la última palabra en materia fiscal dado que representaban a todos, lo que les convirtió en intermediarios natos entre el Estado y los campesinos, con resultados nada halagüeños para estos últimos.

\section{El aprovechamiento de una fuente fiscal en la investigación histórica}

De lo expuesto se deducen los múltiples inconvenientes que presentan los amillaramientos para su utilización como fuente histórica... pero también sus enormes posibilidades. En primer lugar porque, como ya se ha señalado, el nivel de fraude y las distorsiones relativas no fueron las mismas en todas partes. De ahí la necesidad reiteradamente apuntada de insistir en los estudios microespaciales pero, sobre todo, de cruzar las informaciones procedentes de estas fuentes fiscales con otras de distinto origen y características. Y ello no sólo para establecer con mayor precisión los límites de la documentación aquí estudiada, sino para mejorar nuestros conocimientos sobre las sociedades rurales. De hecho, los avances más importantes conseguidos en los últimos años se deben a este tipo de prácticas metodológicas, como ha tenido ocasión de comprobarse en los últimos encuentros verificados sobre el tema de la propiedad y de la explotación agraria ${ }^{2}$. Las conclusiones finales han sido siempre las mismas: afirmación de la pertinencia del recurso sistemático a los amillaramientos con todas las precauciones que se consideren

${ }^{2}$ Se trata especialmente de los dos siguientes: La propiedad de la tierra y los regímenes de tenencia en los siglos XIX y XX. IV Encuentro del Seminario de Historia Agraria (SEHA). Torremolinos, 3-5 de octubre de 1991 y Los espacios rurales y urbanos a través de las fuentes catastrales. UIMP, Valencia, 1-2 de abril de 1992. 
necesarias, primero, y reconocimiento de la necesidad de correlacionar los materiales procedentes de dichos documentos con otros de tipo demográfico, económico, jurídico o socio-político, después. Sobre todo, si se desea lograr análisis más complejos. En esta línea de trabajo se mueven hoy diversos equipos de investigación de historia agraria.

Existen tres ámbitos temáticos en los que los procedimientos descritos resultan particularmente fructíferos:

1. El conocimiento del paisaje agrario. Puede afirmarse que en líneas generales las posibilidades de reconstrucción del mismo a través de los amillaramientos son muy amplias, puesto que suelen incluir cifras concretas sobre la extensión ocupada por los distintos aprovechamientos agrícolas, los pastos, prados y eriales, y las superficies forestales. En una palabra, proporcionan todos los datos necesarios para la elaboración de un mapa de cultivos que materialice espacialmente el uso del suelo. Aunque el detalle de las evaluaciones numéricas requiera alguna rectificación, el panorama global es casi siempre correcto. Huelga decir que se trata de un dato cuyo conocimiento resulta fundamental para establecer las diversas categorías de propietarios, ya que el tipo de cultivo y su mayor o menor intensidad condiciona las fronteras que las separan entre sí, así como, en otro orden de cosas, el tamaño de la unidad de explotación, las formas de tenencia y las estrategias de gestión adoptadas por los terratenientes acomodados en busca de una optimización de sus recursos.

Tal vez convenga aludir en este apartado al ámbito espacial al que están adaptados los textos que estamos analizando, que es siempre el del municipio. Ello plantea una serie de problemas de diversa índole. Geográficos, en primer lugar, ya que las circunscripciones de este origen raramente coinciden con los límites climáticos y orográficos de las distintas comarcas, y económicos en segundo, ya que con mucha frecuencia los grandes patrimonios territoriales se dispersan en los términos de diversos Ayuntamientos, lo que dificulta su reconstrucción. De ahí que hoy hayan aparecido entre los investigadores dos nuevas tendencias al respecto: la puesta a punto de agrupaciones comarcales cuya documentación se estudia conjuntamente -es lo que se ha hecho en Navarra, en el Valle del Guadalquivir o en Extremadura - y la verificación de muestreos a escala provincial; en este caso suelen elegirse municipios ubicados en las áreas geográficas más características - procedimiento seguido en Cataluña, Granada, Valencia y Murcia. La formación de 
equipos ha facilitado la ejecución de estos programas de investigación, que exigen el manejo de un volumen de datos realmente impresionante, accesible hoy gracias a su informatización sistemática.

2. Las estructuras agrarias. Las grandes unidades de análisis en lo que se refiere a este segundo ámbito temático son dos: las estructuras de la propiedad y las de la explotación. Aunque los amillaramientos permiten casi siempre diferenciarlas, los investigadores han centrado tradicionalmente sus esfuerzos en el estudio de las primeras olvidando que las segundas arrojan una imagen bastante más fiel de las jerarquías de clase de la sociedad rural, ya que nos proporcionan una distribución de la tierra más acorde con la realidad. En cualquier caso, hoy parece claro que sin un mejor conocimiento de ambas resulta muy difícil comprender las tendencias a la estabilidad y/o al cambio de las sociedades precitadas; con harta frecuencia, incluso, constituyen las variedades explicativas más importantes al respecto. De ahí que, más allá de las inexactitudes en las cifras globales - perfectamente identificadas y rectificables, como se ha señalado-, el recurso a la fuente fiscal aquí considerada resulta ineludible, ya que el estudio pormenorizado de la misma pone en nuestras manos una masa de informaciones, aproximaciones y «pistas» que por sí solas o con la ayuda de otras fuentes nos va a permitir la caracterización de las estructuras agrarias del área espacial concernida.

Comenzando, pues, por lo referente a la propiedad, existe una primera aproximación relativamente fácil de verificar incluso a un nivel elemental de análisis de los datos: la ubicación del municipio o comarca estudiados en el seno de la dualidad existente entre áreas dominadas por la gran propiedad - latifundista o no-y/o la gran explotación y aquellas otras en las que la unidad de cultivo campesina, pequeña o mediana, resulta hegemónica. En este segundo caso, es fácil deducir la existencia de una agricultura de dominante campesina, mayoritaria a orillas del Mediterráneo a mediados del XIX, como nos recuerda P. Bevilacqua (1989), frente al auge de la gran hacienda trabajada por asalariados, característica de la Europa del Noroeste. Dentro de la propia región andaluza, los resultados de los trabajos de Mata Olmo en su mitad occidental y los de M. González de Molina o M. Martínez Marín en la oriental se han apoyado en gran parte en datos extraídos de los amillaramientos, habiendo conseguido identificar con claridad los caracteres opuestos de estos dos conjuntos subregionales. 
Segundo nivel de aproximación, bastante más complejo: el estudio del reparto de la tierra entre los contribuyentes del término. El punto de partida al respecto puede ser, por un lado, la distinción entre vecinos y forasteros y, por otro, el cálculo del porcentaje que representan los propietarios sobre el total de vecinos, dato este último que requiere la consulta de algún padrón o censo. El conocimiento de ambas cuestiones parece necesario, ya que la presencia mayor o menor de terratenientes que no residen en el término municipal nos permite detectar la existencia de problemas de absentismo y los fenómenos de «colonización» urbana entre otros, mientras que el nivel de acceso a la propiedad del suelo constituye una de las notas fundamentales de las estructuras agrarias y que contribuyen en mayor medida a definir su fisonomía. Además, el distinto grado de acceso a la propiedad territorial constituye un factor decisivo en la articulación de las relaciones sociales.

Pasando así a la distribución de la tierra, propiamente dicha, es preciso admitir que con las fuentes aquí analizadas no es posible conocerla con exactitud rigurosa; en ello reside la gran limitación de las mismas, derivada de la presencia de fraudes importantes en los tramos superiores. Ahora bien, tales prácticas no consiguen en ningún caso alterar el orden de la jerarquía de riqueza, todo lo más atenuar sus diferencias más explosivas. Es aquí donde el recurso a otro tipo de fuentes puede ser útil; así, determinadas actas notariales a las que ya aludimos, que suelen concernir con mayor frecuencia a los grandes propietarios que a los miembros de categorías inferiores y, en las zonas de regadío, a los padrones de acequias. De esta forma se puede proceder a rectificaciones de detalle que resultan sumamente útiles.

Se ha sugerido también la posibilidad de sustituir al individuo como unidad del análisis por el grupo doméstico, ya que en sociedades campesinas la unidad de gestión patrimonial es este último (Martínez Marín, M. y González de Molina, M.: 1992). Esta práctica podría también ayudar a reducir el nivel de ocultación ya que, aunque el cabeza de familia suele ostentar la titularidad jurídica del patrimonio, aparecen con relativa frecuencia situaciones en las que distintos miembros del grupo en cuestión pueden poseer parcelas de tierra que se enumeran separadamente. De esta manera, la acumulación de varias propiedades en una misma familia puede provocar simultáneamente la reducción del tramo de patrimonios territoriales inferiores a una hectárea y la de los patrimonios medios e incluso el reforzar el tramo correspondiente a los 
más grandes. En cualquier caso, las diferencias no suelen ser sustanciales.

Existe un último indicador cuyo cálculo es de utilidad indudable: la cantidad media de tierra per capita, cuyas enormes diferencias a nivel del Estado han servido de base, junto con datos de otra índole, para señalar el dualismo agrario entre la España del Norte y la del Sur, grosso modo. Asimismo, se pueden y deben calcular las extensiones medias poseídas por los agricultores en cada tipo de cultivo. Cuando se ha trabajado a escala regional, como es el caso de Mata Olmo para el Valle del Guadalquivir y yo misma para Murcia, se comprueba que el tamaño medio del patrimonio así como el de la unidad de explotación suele ser el resultado de la evolución histórica, de la calidad del suelo y del aprovechamiento agrario dominante.

Tercer nivel de aproximación: el establecimiento de las distintas categorías de propietarios en función de su nivel de acceso a la tierra. Habitualmente se admite la existencia de tres que corresponden a la pequeña, mediana y gran propiedad, cuya jerarquía suele reflejar con bastante exactitud las grandes divisiones internas de la sociedad rural. Como es lógico, los límites que las separan varían extraordinariamente en función de la calidad del suelo, el tipo de cultivo y/o la mayor o menor intensidad del mismo, datos todos ellos que aparecen casi siempre en los amillaramientos, de ahí su utilidad en este apartado. A señalar, además, que la imagen de diversidad que se deduce del conocimiento de estos aspectos puntuales ha ayudado a superar los esquemas preestablecidos sobre la definición de las tres categorías en cuestión. Hoy se huye de las caracterizaciones cuantitativas centradas exclusivamente en el número de hectáreas atribuible a cada una de ellas, prefiriéndose conceptos más amplios y flexibles.

En esta línea, la pequeña propiedad no se establece únicamente por su extensión - dato estadístico- sino por un dato económico: su incapacidad para cubrir las necesidades del mantenimiento familiar, lo que obliga a los titulares de la misma a la búsqueda de trabajo fuera de la explotación. Y otro tanto ocurre con las de tipo mediano y grande, definidas, a su vez, la primera por la suficiencia de la fuerza de trabajo propia para cubrir las tareas agrícolas, aunque estacionalmente se requiera el recurso a la mano de obra asalariada, y la segunda por la necesidad sistemática de acudir a esta última. A partir de estos presupuestos, la constitución de los diferentes tramos de propiedades y/o 
propietarios se convierte en un ejercicio útil que posee mayor alcance y es más ilustrativo que el mero cálculo de los índices de concentración, como el de Gini por ejemplo.

La virtualidad del esfuerzo en este sentido es a la vez económica y social. Económica, en primer lugar, porque permite averiguar el peso relativo de los tres tipos de propiedad indicados, dato fundamental tanto para caracterizar las estructuras agrarias como para dilucidar a cual de ellas correspondió un mayor protagonismo en los diversos procesos de cambio agrario. Así, en lo que concierne a las de tamaño reducido hoy sabemos que, aunque la cantidad de suelo globalmente ocupada por ellas suele ser baja, cuando se suma con la correspondiente a las parcelas llevadas en arrendamiento o en aparcería se obtienen cifras sorprendentes por su elevación. El mejor ejemplo lo constituyen, tal vez, determinadas regiones mediterráneas, en las que las pequeñas explotaciones propias o arrendadas ocupan un porcentaje mayoritario del suelo cultivado en todas las comarcas de regadío, por lo que podría hablarse de una «vía campesina» de penetración del capitalismo en las mismas (Pérez Picazo, M.T.: $1995^{\mathrm{a}}$ ). Y sociales, en segundo, porque el conocimiento de los porcentajes de tierra que corresponden a los principales estratos en que se divide el campesinado ayuda a precisar, como se ha insinuado, las estructuras de clase de la sociedad rural. Dado que parecen superadas las tendencias a establecer unas fronteras demasiado rígidas entre los tres grupos, las informaciones aproximativas de los amillaramientos nos convienen perfectamente.

Pero además, dichas informaciones son susceptibles de otras formas de aprovechamiento siempre referibles a la distribución de la tierra y a la fisonomía de los titulares de la misma:

a) La ubicación física de las parcelas correspondientes a la pequeña, mediana y gran propiedad en relación con los distintos sistemas de cultivo y con la calidad del suelo cultivable. Se trata de averiguar si el porcentaje que corresponde a cada una de ellas en los diferentes medios agrícolas es similar o si, por el contrario, los grandes terratenientes controlan determinadas producciones y/o las mejores tierras. Mata Olmo (1987), por ejemplo, ha mostrado en la Campiña Bética cómo los latifundios identificados en los amillaramientos-se asientan preferentemente en los suelos profundos del fondo del Valle del Guadalquivir - «suelos vérti$\cos »$ - fértiles, pero que requieren una fuerte inversión en trabajo, 
mientras que la pequeña propiedad se refugia en las pendientes de la sierra, de suelos menos fértiles pero más fáciles de trabajar. En otro espacio muy distinto, la cuenca del Segura, la afortunada conservación de amillaramientos y padrones de acequias para las mismas fechas en el municipio de Calasparra, me ha permitido descubrir un magnífico ejemplo de geografía social desequilibrada. Ello se debe al desigual reparto de la propiedad existente en las dos zonas de regadío de las que dispone el municipio en cuestión, una acondicionada con las abundantes dotaciones hídricas del Segura y la otra con las muchas más escasas del río Argos; en la primera, el $85 \%$ del suelo pertenecía en 1860 a cuatro propietarios mientras que en la segunda el $78,5 \%$ se distribuía entre explotaciones de tamaño pequeño o mediano ${ }^{3}$. Un trabajo parecido ha sido realizado por los historiadores catalanes citados y por algún otro como R. Congost $(1990,1991)$ los cuales, mediante la integración de datos procedentes de distintas fuentes, han conseguido reconstruir el paisaje social de los municipios y comarcas objeto de investigación.

b) Identificación de la elite de grandes terratenientes, cuya posición relevante aparece siempre en los amillaramientos por muy elevado que sea el grado de ocultación. Una vez enumerados sus miembros, es necesario, además, buscar informaciones complementarias, como la que proporcionan los listados de la Contribución Territorial y los Inventarios post mortem, muy fiables en estos años, por lo que nos ayudan a determinar las dimensiones exactas de los patrimonios más importantes y la composición de los mismos. En los trabajos cuyo universo de análisis es el comarcal, el esfuerzo no es muy grande y compensa por las precisiones que introduce. El segundo paso debe dirigirse, más allá del esfuerzo puramente identificativo y cuantitativo, a ubicar socialmente la elite en cuestión, ya que los cambios sucesivos acaecidos en su seno constituye uno de los indicadores más importantes de la evolución socio-económica experimentada entre la Revolución liberal y la Guerra Civil.

c) La movilidad de la tierra, cuyo índice más claro es el volumen de compraventas a lo largo de un período determinado. En estos últimos años se están comenzando a utilizar con tal fin los Apéndices de los Amillaramientos, pese al notable trabajo que supone la cuantificación de todas las operaciones (Sabio Alcutén, A: 1992; García Sexto A.:

3 Amillaramiento de 1863, Calasparra (Archivo Municipal). Padrones de las Acequias de Rotas, Berberín y Esparragal (río Segura) y de las siete que derivan el agua del Argos (Heredamiento de Calasparra) 
1988; Colomé, J. y Saguer, E.: 1992). El primero de los historiadores citados ha centrado su investigación en el municipio aragonés de Cariñena para el período 1886-1934. Entre sus principales conclusiones destaca el carácter «forzado» que atribuye a la adquisición de pequeñas parcelas por parte de los cultivadores modestos, que se encontraban ante la necesidad perentoria de recomponer sus explotaciones para que fuesen viables tras las subdivisiones impuestas por los sistemas de sucesión igualitarios. ¿Se podría extender este modelo a campesinos pertenecientes a estratos más altos o ubicados en otro tipo de comarcas? Según parece, los comportamientos de los pequeños viticultores catalanes no eran muy distintos: E. Saguer calcula que en los municipios por él estudiados las transacciones protagonizadas por aquéllos equivalieron a un $27 \%$ de la superficie amillarada. En ambos casos, pues, no habría tenido lugar un proceso de desposesión del pequeño campesinado, sino una sustitución sucesiva de los titulares de las parcelas por otros individuos procedentes del mismo grupo social. Semejante evolución contrasta con la acaecida en la mayor parte de las huertas levantinas en el siglo XIX (Calatayud, S.: 1988; Millán García-Varela, J.: 1992, 1995).

En lo que se refiere a las estructuras de la explotación, la importancia de su reconstrucción ya ha sido señalada; por consiguiente, de lo que ahora se trata es de enumerar qué tipo de informaciones relativas a las mismas figuran en los amillaramientos y cómo se les puede sacar partido. En mi opinión podrían resumirse en tres, dos de índole cuantitativa y una tercera cualitativa: la extensión de cada una de las parcelas cultivadas por «colonos» - dato que nos permite averiguar el alcance absoluto y relativo de la gestión indirecta- así como los nombres de los mismos; la composición de las unidades de cultivo, puesto que al lado de dichos nombres suele figurar un listado de las tierras que trabajan los individuos que las llevan, y, en determinado número de casos pero no siempre, la forma de tenencia.

El primer grupo de datos propicia una vez más el establecimiento de las diversas categorías de explotadores y explotaciones con los mismos criterios de clasificación que se han utilizado en lo concerniente a la propiedad. Es decir, la agrupación tripartita en pequeños-as, medianos-as y grandes. Todo lo dicho en aquel caso puede repetirse en este. En cuanto al segundo bloque, requiere cierta atención ya que a partir de los elementos que proporciona podemos llegar a conocer la unidad de

$$
-303-
$$


cultivo real. Y ello porque en la mayoría de las comarcas agrícolas dichas unidades suelen estar integradas por tierras propias y arrendadas en distinta proporción y, asimismo, por parcelas ubicadas en zonas de cultivo diferente. Así, en los secanos mediterráneos coexisten explotaciones cerealeras con plantíos (arbolado y/o viñedo), separados o en régimen de colture promiscue; en Galicia suele incluirse el acceso al monte de una u otra forma, etc. Sólo se exceptúan de esta regla general las zonas de monocultivo, ya se trate de las comarcas cerealeras de Castilla la Vieja, de las vitícolas de Cataluña, Valencia y Murcia o de las huertas levantinas. Pero en las áreas de viñedo los campesinos poseen con cierta frecuencia alguna parcela sembrada de trigo en las inmediaciones del núcleo urbano, con posibilidad de riego o sin ella —es el caso de algunos municipios catalanes, de los del Valle del Vinalopó o de Jumilla-Yecla. Y en cuanto a los huertanos, la tendencia a la intensificación de los cultivos - característica de la segunda mitad del XIX- les permitía intercalar dicha gramínea en las rotaciones con plantas hortícolas; la práctica figura a veces en los propios amillaramientos, como sucede en los municipios murcianos de Cehegín y Mula. ${ }^{4}$

Resumiendo, estos agregados de parcelas contiguas o dispersas deberían ser consideradas como los grandes protagonistas de la organización agraria. Su reconstrucción estadística y la descripción de los diversos elementos que los componen son perfectamente factibles mediante el recurso sistemático a los amillaramientos. Además, como suele incluirse en el listado el posible acceso a una vivienda en el medio rural o en el núcleo de población correspondiente, se puede deducir sin mayor problema si el campesino explotador reside o no junto a sus tierras. El panorama queda perfilado con el manejo de otros dos tipos de fuentes que complementan a la precitada: los Padrones de Vecinos y, cuando existen, los de Colonos. La aplicación de este sistema al secano del Campo de Cartagena y Murcia ha permitido, por ejemplo, esbozar las grandes líneas del poblamiento de ambos en el siglo XIX, caracterizado grosso modo por la existencia de pueblos poco numerosos y de tamaño reducido - entre 500 y 1.800 habitantes - pero, sobre todo, por la relativa densidad de viviendas aisladas. Conclusión sorprendente en un medio ubicado en el Sureste climático y que solo cobra sentido cuando se conocen los complejos sistemas de aprovechamiento hidráulico puestos a

${ }^{4}$ Amillaramientos de 1860, Cehegín y Mula (Archivo Municipal). Padrones de las acequias del Argos y del Quípar (Asociación de Regantes de Cehegín) 
punto por los habitantes de la zona, apoyado esencialmente en el acondicionamiento de una extensa red de boqueras a partir de las numerosas ramblas (Pérez Picazo, M.T.: 1995b). Además, hemos podido determinar que el $78,6 \%$ de los vecinos eran aparceros («terrajeros») y el resto jornaleros, y que sus explotaciones incluían tierras propias - del 20 al 40\% como media - y otras llevadas en aparcería. Prácticamente todos reunían parcelas dedicadas al cultivo de los cereales (un $66,7 \%$, de las que la mitad eran de su propiedad) con otras plantadas de olivo $(28,5 \%)$ y vid (5,8\%), estas dos últimas casi siempre de terratenientes forasteros (Pérez Picazo, M.T.; Martínez Carrión, J.M.; Pérez de Perceval, M.A.: 1996).

Por último, los datos referentes a la posesión de ganado mayor o menor figuran en casi todos los amillaramientos de las diversas regiones. Obviamente, no intento deducir de este hecho que el recuento sea muy fiable (hay otras fuentes para llegar a este tipo de estimaciones), sino que la importancia relativa de dicho tipo de bienes resulta muy ilustrativa en la descripción de la fisonomía de las unidades de cultivo. Primero, porque constituyen un buen indicador del nivel de acomodo de los titulares de las mismas, especialmente en lo que se refiere al ganado de tiro, cuya fuerza de trabajo era indispensable en unas agriculturas mayoritariamente «orgánicas». Y después, porque la presencia generalizada de rebaños de cabras y ovejas en unos casos o de reses vacunas en otros apunta hacia la presencia dominante del elemento pecuario en la actividad agraria. Es lo que sucede, por ejemplo, en áreas montañosas, como la compartida por Almería, Granada, Noroeste de Murcia y de Albacete, en cuyos amillaramientos es frecuente la inclusión de vecinos sin más propiedad que una casa en el núcleo de población y un pequeño rebaño más, en determinadas ocasiones, una parcela de reducido tamaño en la aureola de agricultura intensiva del núcleo en cuestión. En estos casos la gran propiedad, sea cual fuere su fisonomía y dedicación, se ubica en los vastos espacios intercalares, raramente en las inmediaciones del centro municipal.

Por último, el estudio de las formas de tenencia constituye tal vez el aspecto de las estructuras de la explotación que presenta una información más desigual en los amillaramientos, tanto a escala suprarregional como en el interior de la misma región. Sin motivo aparente, en efecto, algunos incluyen referencias al uso del arrendamiento, aparecería o enfiteusis, mientras que otros permanecen «mudos» al respecto. Pero, en los casos en los que el dato aparece, su presencia proporciona al in- 
vestigador una ayuda inestimable, ya que le permite no sólo averiguar cual es el tipo de contrato dominante sino traducirlo en porcentajes, por muy aproximativos que resulten.

3. El estudio de la familia y del hogar campesino. Las aportaciones de un colectivo de investigadores catalanes (Ferrer, Ll.: 1987), navarros (Erdozain, P.: 1995; Mikelarena, F.: 1993) y mallorquines (Moll, I.: $1992,1993)$ han puesto de manifiesto la necesidad de integrar en el estudio de las estructuras agrarias el tema de la familia campesina. Lo cual supone una vez más el cruce de informaciones procedentes de múltiples tipos de fuentes: censos de población, amillaramientos, documentación notarial, libros de bautismos y defunciones de los Archivos Parroquiales, Registro Civil, etc. Este nuevo enfoque tiende a convertir las unidades de producción familiar en un núcleo central del análisis de historia agraria. Ello permitiría, en opinión de sus defensores, articular dos líneas de investigación que hasta ahora han funcionado en paralelo: la que desde el ámbito de la demografía histórica estudia la familia y el hogar como componente básico de la estructura social y la que desde la historia económica viene centrando su atención en los procesos de cambio agrícola asociados a la expansión del mercado.

En esta línea, el uso de los amillaramientos aporta una masa de datos que pueden ser decisivos, ya que las características de muchos de ellos influyen de mil maneras en las condiciones de la reproducción familiar. Así, la extensión de la unidad de explotación, la composición de la misma, el sistema de cultivo - ¿orientado a la subsistencia o al mercado?-, las formas de tenencia, etc. Elementos todos ellos que en absoluto se pueden obviar, al igual que sucede con las diferentes maneras de ocupación del espacio agrario, cuando se pretende profundizar en el análisis del comportamiento económico de las unidades familiares. Se podría concluir diciendo que el objetivo final de estos laboriosos análisis en que se integran datos de tan diversa procedencia consistiría en sacar a la luz la red de relaciones entre el grupo doméstico, la propiedad de la tierra y los procesos de trabajo.

\section{Conclusión}

El balance que venimos de realizar no puede ser homogéneo ni definitivo, sobre todo conociendo las enormes diferencias regionales, co- 
marcales y municipales al respecto. Pero, pese a todo, siempre que sea posible manejar los amillaramientos, su uso sigue siendo necesario; no existe otra documentación que, como ésta, permita de forma global y sintética la reconstrucción aproximada del reparto de la propiedad y de la fisonomía de la unidad de explotación para la segunda mitad del siglo XIX.

Ello no obsta para que se reconozcan las limitaciones de la fuente, lo que supone para el investigador un esfuerzo supletorio de depuración de los datos y, siempre que sea posible, el contraste de los mismos con otros de diferente origen heurístico. Creo haber mostrado en las páginas precedentes que el esfuerzo combinado de un denso grupo de especialistas de historia agraria en este sentido ha conseguido a lo largo de la última década, por un lado, extraer el máximo provecho posible de una documentación rica pero de difícil manejo, y, por otro, guardar las debidas precauciones para evitar la aceptación acrítica de las informaciones conseguidas. Gracias a ellos hoy sabemos un poco más de la sociedad rural española del siglo XIX.

\section{BIBLIOGRAFÍA}

Alzina i Mestre, J. (1993): Població, terra i propietat a la comarca de Llevant de Mallorca (segles XVIII, XIX y XX): municipis d'Artá, Capdepera $i$ Son Servera. Ayuntamiento de Artá.

Artola, M. (1986): La Hacienda en el siglo XIX. Progresistas y moderados. Madrid, Alianza Editorial.

Bernal, A. M. y Drain, M. (1975): Les campagnes sevillannes aux XIX ${ }^{e}-X X^{e}$ siècles. Rénovation ou stagnation. París, Casa de Velázquez.

BEVILACQUA, P. (a cura di) (1990): Storia dell'agricultuta italiana in etá contemporanea. II. Uomini e classi. Venecia, Marsilio Editore.

Calatayud, S. (1989): Capitalismo agrario y propiedad campesina. La Ribera del Xúquer, 1860-1930. Valencia, Instituto Alfonso el Magnánim.

Comín, F. (1988): Hacienda y economía en la España contemporánea (1800-1936). 2 vols, Madrid, I.E.F.

Congost, R. (1991): «Enfiteusis y pequeña explotación campesina en Cataluña, siglos XVIIIXIX». En Saavedra, P. y Villares, R. (eds.): Señores y campesinos en la Península Ibérica, s. XVIII-XX. 1. Campesinado y pequeña explotación. Barcelona, Crítica, pp. 6388.

- (1990): Els propietaris i els altres. La regió de Girona, 1768-1862. Vich, Eumo Editorial.

CruZ Villalón, J. (1980): Propiedad y uso de la tierra en la Baja Andalucía, siglos XVIII$X X$. Madrid, MAPA.

ERDOZAIN, P. (1991): «Relaciones entre el sistema sucesorio, la estructura del hogar y la propiedad de la tierra en la Navarra media occidental». Comunicación presentada a la IV Reunión del Seminario de Historia Agraria (SEHA).

$$
-307-
$$


ERDozAIN, P. (1995): «Familia, patrimonio y proceso de trabajo en la Navarra media occidental a finales del siglo XIX». Noticiario de Historia Agraria, n. ${ }^{\circ}$ 5, p. 25-42.

FERRER, Ll. (1987): Pagesos, rabassaires $i$ industrials en la Catalunya Central (segles XVIII-XIX. Barcelona, Pub. Abadía de Montserrat.

FErRer, Ll.; Segura, A. y SuAu, J. (1985): «Confecció dels Amillaraments i poder local: els municipis del Plá de Barcelona, 1851-1874». III Jornades d'Estudis Historics Locals, Instituto de Estudios Baleáricos, Palma de Mallorca, pp. 145-158.

Fontana, J. (1977): La Revolución liberal. Política y Hacienda (1833-1845). Madrid, I.E.F.

FuENTES Quintana, E. (1961): «Los principios del reparto de la carga financiera en España», Revista de Derecho Financiero, n. ${ }^{\circ} 41$, pp. 161-185.

- (1990): Las reformas tributarias en España. Barcelona, Crítica.

GARCÍA SEXTO, A. (1988): «Transformacions na propedade da terra no Concello de Santiago (1906-1941)». En R. Villares (ed.): Donos de Seu. Estudios de Historia Agraria de Galicia. Barcelona, Sotelo Blanco, pp. 325-365.

Garrabou, R. (coord.) (1992): Propiedad y explotación campesina en la España contemporánea. Madrid, MAPA.

Garrabou, R.; Pujol, J.; Colomé, J. y Saguer, E. (1992): «Estabilidad y cambio en la explotación campesina (Cataluña, s. XIX y xx)». En Garrabou (ed.), pp. 15-23.

GONZÁLEZ DE MOLINA, M. (1993): «La funcionalidad de los poderes locales en una economía orgánica». Noticiario de Historia Agraria, n. ${ }^{\circ} 6$, pp. 9-25.

Grupo de Historia Agraria de Murcia (1992): «La propiedad de la tierra en los regadíos del Segura durante los siglos XIX y XX». En Garrabou (ed.), pp. 15-191.

Grupo de Investigación del Instituto Gerónimo de Uztáriz de Pamplona (1992): «La propiedad privada en Navarra a fines del siglo XIX». En Garrabou (ed.), pp. 93-156.

GutiérRez PoCH, M. (1992): «La propiedad de la tierra en una comarca de Cataluña, l'Anoia en la segunda mitad del XIX». En Garrabou (ed.), pp. 293-325.

Laguna Platero, A. y MARTínez Gallego, F. (1995): «Moderados, progresistas y unionistas (1844-1868)», En M. Chust (dir.) Historia de la Diputación de Valencia. Valencia, Diputación de Valencia, pp. 107-174

MARTÍNEZ MARÍN, M. (1995): Revolución liberal y cambio agrario en la Alta Andalucía. Granada, Universidad de Granada.

Martínez Marín, M. y GonZÁlez de Molina, M. (1992): «Notas sobre propiedad y explotación como variables explicativas en la historia de las comunidades campesinas andaluzas». En Garrabou (ed.), pp. 351-379.

Mata Olmo, R. (1987): Pequeña y gran propiedad agraria en la Depresión del Guadalquivir. 2 vols. Madrid, MAPA.

MATa Olmo, R. y Romero GonZÁlez, J. (1988): «Fuentes para el estudio de la propiedad agraria en España (s. XVIII-XX)». Agricultura y Sociedad, n. ${ }^{\circ} 49$, pp. 209-293.

Millán GARCía-VARELA, J. y CALATAYUd, S. (1992): «Formas de explotación y mercados de trabajo en la agricultura valenciana de regadío (1800-1930)». En Garrabou (ed), pp. 325-351.

MiLlán, J. (1991): «Gran propiedad y pequeña explotación en el desarrollo del capitalismo agrario valenciano. El Bajo Segura, 1800-1950». En Saavedra, P. y Villares, R. (eds.): Señores y campesinos en la Península Ibérica, siglos XVII-XX. 1. Campesinado y pequeña explotación. Barcelona, Crítica, pp. 7-34.

Moll Blanes, I. (1987): La estructura familiar del campesinado de Mallorca, 1824-1827. En La familia en la España Mediterránea (siglos XV-XIX). Barcelona, Crítica, pp. 212257.

- (1995): «Las redes familiares en las sociedades rurales». Historia Social, n. ${ }^{\circ} 22 ; \mathrm{pp}$. 125-143.

Moll, I. y Mikelarena, F. (1993): «Elementos para el estudio de las sociedades agrarias: 
de los procesos de trabajo al ciclo de vida». Noticiario de Historia Agraria, n. ${ }^{\circ}$ 5, pp. 25-42.

MoReno PeÑa, J.L. (1992): Gran propiedad rústica en Burgos. Burgos, Caja de Ahorros Municipal.

OBIOL MENERO, E. (1988): Terra i propietat a la Borriana tarongera del segle XIX. Les transformacions rústiques de Polo de Bernabé. Burriana, Papers.

PÉRez PICAZO, M. T. (1991): «Riqueza territorial y cambio agrario en la Murcia del siglo XIX. Aproximación al estudio de una contabilidad privada». Agricultura y Sociedad, n. ${ }^{\circ} 66$, pp. 39-94.

- (1995a): «Pequeña explotación y consolidación del capitalismo en las agriculturas mediterráneas». En Morilla Critz (ed.): California y el Mediterráneo, historia de dos agriculturas competitivas. Madrid, MAPA.

- (1995b): «Tecnología hidráulica y estructuras sociales en los campos costeros de la región murciana». En González Alcantud, M. (coord.): El agua. Mitos, ritos y realidades. Madrid, Anthropos.

Pérez Picazo, M. T.; MarTínez Carrión, J. M. y Pérez de Perceval, M. A. (1996): Grupos sociales, estructura productiva y explotación agraria en el sureste español en los siglos XIX y XX. Informe final del Proyecto de Investigación. PB 91-6947/29/4/94 (DGCYT).

Pro Ruz, J. (1992): Estado, geometría y propiedad. Los orígenes del Catastro en España. Madrid, Centro de Gestión Catastral y Cooperación Tributaria.

- (1993): «Las elites locales y el poder fiscal del Estado en la España contemporánea». Les elites locales et l'Etat dans l'Espagne moderne, XVI ${ }^{e}-X I X^{e}$ siècles. París, Ed. del C.N.R.S., pp. 283-295.

- (1994): «Fraude, statistique et pouvoir dans l'Espagne liberale (1840-1868)». Revue d'Histoire moderne et contemporaine, abril-junio, pp. 253-269.

- (1996): «Las contribuciones de producto «antiguas» de 1845». Hacienda Pública Española, Monográfico sobre La reforma fiscal de Mon-Santillán ciento cincuenta años después, pp. 119-135.

ROMERo GONZÁlez, J. (1983): Propiedad agraria y sociedad rural en la España mediterránea. Madrid, MAPA.

SABIo AlCUTÉn, A. (1992): «Las compraventas de tierra, una faceta de la integración mercantil forzosa del pequeño campesino. Cariñena, 1886-1936». Noticiario de Historia Agraria, n. ${ }^{\circ}$ 4, pp. 315-335.

SAGUER, E. (1992): «La evolución de la propiedad en el Baix Empordá (Girona), 1860-1950». En Garrabou (ed.), pp. 261-293.

SÁNCHEZ MARROYO, F. (1993): Dehesas y terratenientes en Extremadura: la propiedad de la tierra en la provincia de Cáceres en los siglos XIX y XX. Cáceres, Asamblea Regional de Extremadura.

SEguRA, A. (1988): «La reforma de Mon (1845) y los amillaramientos en la segunda mitad del XIX». El Catastro en España, 1714-1906. I. De los Catastros del siglo XVIII a los Amillaramientos de la segunda mitad del XIX. Madrid, Centro de Gestión Catastral y Cooperación Tributaria, pp. 113-135.

(1993): Burguesía i propietat de la terra a Catalunya en el segle XIX. Les comarques barcelonines. Barcelona, Curial.

SUAU, J. (1991): El mon rural mallorqui (segles XVIII-XIX). Barcelona, Curial.

VILLARES, R. (1982): La propiedad de la tierra en Galicia, 1500-1936. Madrid, Siglo XXI. 
RESUMEN: Fuentes fiscales e historia agraria. El debate en torno de las posibilidades heurísticas de los amillaramientos. El objetivo del presente artículo consiste en una breve reflexión sobre las posibilidades que ofrece como fuente histórica la documentación generada por una de las figuras tributarias surgidas en 1845: la Contribución de Inmuebles, Cultivo y Ganadería. El tema ha sido objeto de cierto debate, por lo que una parte del trabajo se centra en el análisis de los argumentos a favor y en contra, mientras que en el resto del mismo se exponen las principales aportaciones conseguidas recientemente por un grupo de especialistas de Historia Agraria utilizando, con las debidas precauciones, la documentación indicada, esencialmente los Amillaramientos.

PALABRAS ClAVE: Reforma Fiscal. Contribución de Inmuebles. Cultivo y Ganadería. Investigación de Historia Agraria. Amillaramiento.

RÉSUMÉ: Sources fiscales et histoire agraire. Le debat sur les posibilites heuritiques des amillaramientos. L'objetif du présent article consiste á une bréve reflexion sur les posibilités qui offre comme source historique la documentation generée par un procedé fiscal sorti de la Reforme de 1845: la Contribution d'Inmeubles, Culture et Elevage. La sujet a donné lieu á un certain débat; donc, une premiére partie de mon travail est consacré á une analyse des argumentations pro et contra, tandis que dans la deuxiéme on expose les principaux progrés de nos connaissances sur l'histoire agraires dans ces derniéres années, reussis grâce aux recherches d'un groupe d'especialistes qu'ont utilisé de façon systematique, mais avec des precautions, les Amillaramientos.

MoTS CLÉ: Reforme Fiscal. Contribution d'Inmeubles. Culture et Elevage. Recherche Historique Agraire. Amillaramiento.

ABSTRACT: Financial sources and agrarian history. The debate around the heuristic choices of the amillaramientos. The aim of this article consist in a brief reflexion about the possibilities what the documentation produced by one of the taxes emerged in 1845 offers as historical source; property agriculture and cattle resing tax. The subjet has produced certain debate, consequently some part of this article is focused on the analysis of arguments in favour and against, the rest of the same article is included the main contributions recently achieved by means of an specialist group about Agrarian History making use of sefety mesures set by the documentation, mainly the Amillaramientos (agrarian census).

Key words: Fiscal Reforme. Tax on Urban Property. Agriculture and Cattle. Agrarian issues. Amillaramiento. 